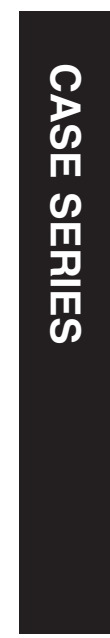

${ }^{1}$ Ophthalmology Unit NESMOS Department, S.Andrea Hospital, Rome, Italy

2DAl Testa/Collo, Azienda Policlinico Umberto I, University of Rome 'Sapienza', Rome, Italy

Correspondence: $S$ Abdolrahimzadeh, Ophthalmology Unit, DAI Testa/Collo, University of Rome 'Sapienza', Azienda Policlinico Umberto I, Viale del Policlinico 155, Rome 00161, Italy Tel: +39 06 49975348; Fax: +3906 49975304 . E-mail: solmazzadeh@ gmail.com

Received: 19 January 2014 Accepted in revised form: 27 March 2014 Published online: 25 April 2014

\section{Intravitreal bevacizumab in the successful management of choroidal metastases secondary to lung and breast cancer unresponsive to systemic therapy: a case series}

$\checkmark$ Fenicia', S Abdolrahimzadeh², G Mannino", S Verrilli ${ }^{1}$, M Balestrieri ${ }^{1}$ and SM Recupero ${ }^{1}$

\begin{abstract}
Purpose Management of choroidal metastases is commonly with systemic chemotherapy; however, if tumours are refractory to treatment and vision is endangered, local therapy modalities are feasible. A novel option is the use of intravitreal bevacizumab. This report presents three cases of choroidal metastatic tumours secondary to lung and breast cancer treated with intravitreal bevazizumab.

Patients and methods Three patients with choroidal metastases secondary to lung and breast tumours were treated at the Ophthalmology Unit, University of Rome 'Sapienza', S.Andrea Hospital from January 2009 to August 2012. All patients developed vision loss with diagnosis of chorioidal metastasis during systemic chemotherapy. Off label intravitreal bevacizumab treatment was performed with two $1.25 \mathrm{mg}$ injections in two patients and four injections in one patient at 30-day intervals.

Results Vision improved, subretinal fluid resolved, and choroidal tumour regression was obtained in all cases. Follow-up was 6,9 , and 12 months and there were no

by preventing vision loss and improving the quality of life of patients.

Eye (2014) 28, 888-891; doi:10.1038/eye.2014.96; published online 25 April 2014

Introduction

Management of choroidal metastases is commonly with systemic chemotherapy; however, if choroidal lesions arise or enlarge during therapy then local treatment modalities such as external beam radiotherapy, plaque brachytherapy, transpupillary thermotherapy, and photodynamic therapy are employed. ${ }^{1-5}$ Bevacizumab is a full-length recombinant humanized monoclonal antibody against all forms of vascular endothelial growth factor A. ${ }^{6}$ A few recent case reports have described the management of metastatic choroidal lesions through intravenous and/or intravitreal administration of bevacizumab with encouraging results. ${ }^{7-12}$

We employed intravitreal bevacizumab in the management of three patients with primitive tumours of the lung and breast who developed choroidal metastases during chemotherapy. The study was approved by the Local Ethics Committee.
\end{abstract} complications related to treatment. Conclusions Intravitreal bevacizumab administration represented an efficacious therapeutic option with rapid effect in the treatment of choroidal metastatic tumours unresponsive to systemic therapy. It can have a role in the management of these tumours
Case reports

Case A

A 39-year-old woman presented to our department complaining of distorted vision in the left eye (LE) since 1 month. Two years 
previously she had undergone bilateral mastectomy and chemotherapy for poorly differentiated invasive carcinoma of the breast. She was on treatment with tamoxifen. Best corrected visual acuity (BCVA) was $20 / 50$. Fundoscopy showed a large choroidal mass in the superior sector (Figure 1a). Fluorescein angiography (FA), B-scan echography and optical coherence tomography (OCT) were performed (Figures $1 \mathrm{~b}-\mathrm{d}$ ). Informed consent for the off label intravitreal use of bevacizumab was obtained and two $1.25 \mathrm{mg}$ injections at 30-day intervals were administered. Fifteen days after the second injection, BCVA improved to 20/25 and regression of the mass was observed (Figures 1e-h). At 6 months, BVCA was 20/20 after which the patient developed multiorgan metastases and died.

\section{Case B}

A 36-year-old woman presented to our department for rapidly decreased vision in the LE since 1 month. She was on treatment with gemcitabine and cisplatin for invasive papillary lung adenocarcinoma diagnosed 6 months previously. BCVA was finger counting. Fundoscopy showed two large choroidal masses in the superior and supero-temporal quadrant with neuroepithelial macular detachment. FA, B-scan echography and OCT were performed. Informed consent was obtained and two $1.25 \mathrm{mg}$ intravireal bevacizumab injections were administered at 30-day intervals. BCVA improved to 20/25 fifteen days after the second injection. Regression of the choroidal masses and neuroepithelial detachment was observed. Based on results the oncologist modified systemic therapy to carboplatin, taxol and intravenous bevacizumab. At 9 months BCVA was stable. The patient was hereafter lost to follow-up.

\section{Case C}

A 54-year-old woman presented to our department for routine ophthalmological examination. She had been diagnosed with breast cancer 16 years previously and had a history of lung and bone metastases treated with several cycles of chemotherapy. She was on treatment with docetaxel since 4 months. BCVA was 20/25 in the LE. Fundoscopy showed a choroidal mass in the superotemporal sector. FA, B-scan echography and OCT were performed. Informed consent was obtained and four $1.25 \mathrm{mg}$ intravitreal bevacizumab injections, at 30-dayintervals, were administered. The choroidal mass regressed after four injections. BVCA improved to 20/20 at 30 days and remained stable for 12 months at which time the patient had systemic progression of disease and deceased.

\section{Discussion}

Treatment of choroidal metastases is usually palliative. The aim of therapy is to restore or maintain visual function and improve the quality of life of patients as far as possible.

Radiotherapy is the standard treatment for choroidal metastases. Lesion regression with external beam radiotherapy has been shown in $85-93 \%$ of cases but
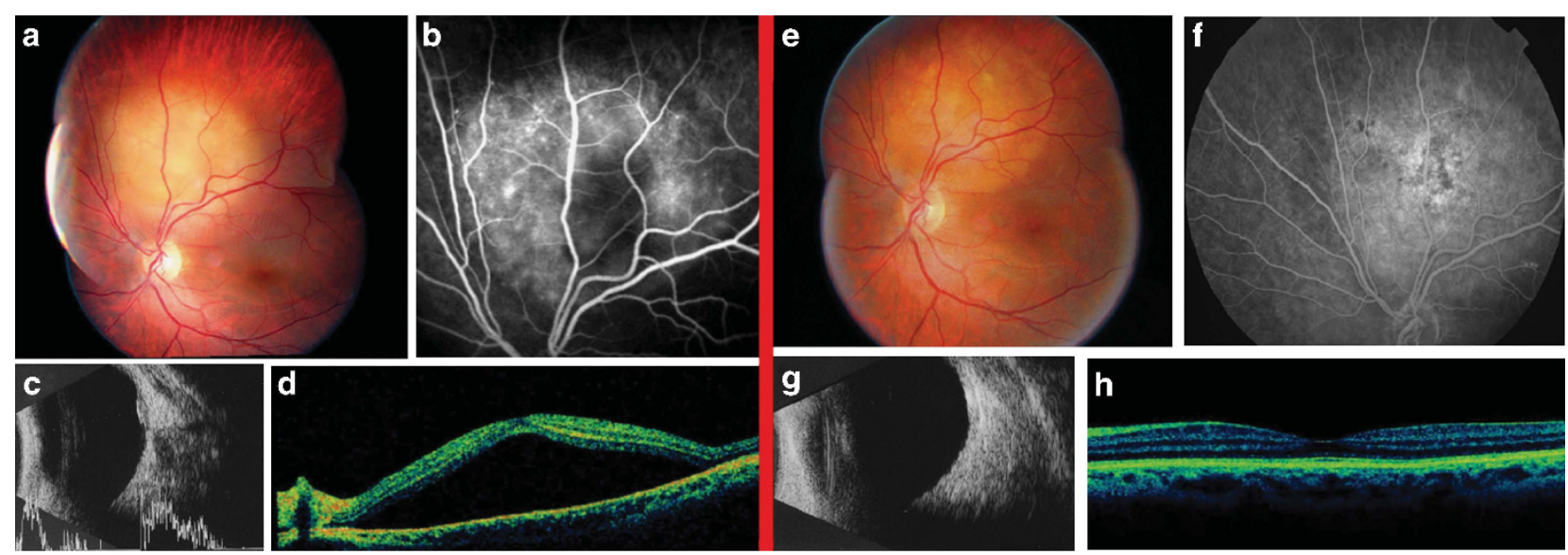

Figure 1 Regression of choroidal metastasis after intravitreal bevacizumab (Case A). Pre-treatment (left images): (a) Fundus photograph shows yellow mass in the superior sector close to the optic disc, (b) FA demonstrates hypofluorescence due to masking effect with signs of leakage, (c) B-scan echography shows a medium-high reflective choroidal mass above the optic disc, (d) OCT demonstrates serous detachment of the neuroepithelium. Post-treatment (right images): (e) Fundus photograph shows regression of the choroidal mass, (f) FA demonstrates pigmentary changes with hypo-hyperfluorescent areas and signs of scarring due to flattening of the mass, (g) B-scan echography shows regression of the mass, (h) OCT demonstrates resolution of the serous detachment. 
radiotherapy-induced complications such as cataracts, exposure keratopathy, iris neovascularization, radiation retinopathy, and papillopathy have been reported in $12 \%$ of cases. Furthermore, the principle setback in patients with metastatic disease is the need for repeated daily treatments. ${ }^{4,13}$ Plaque brachytherapy allows the delivery of radiation directly to the choroid and has shown regression of metastases in up to $94 \%$ of patients with a lower rate of radiotherapy-induced complications. ${ }^{14}$ However, the main disadvantage is the need for surgical procedures to place and remove the plaque. In order to avoid complications of radiation radiotherapy, some authors have employed transpupillary thermotherapy with the infrared diode laser. This method has been used successfully in solitary lesions with thickness of less than $3.5 \mathrm{mms}$ and minimal subretinal fluid. ${ }^{15}$ A recent study reported successful management of choroidal metastases with photodynamic therapy. There was stabilization or improvement of visual acuity and only minor intraretinal haemorrhage related to treatment in seven of eight eyes treated. ${ }^{5}$ The advantage of this treatment is the avoidance of long-duration radiotherapy, hospitalization, or surgery.

The inhibition of tumour-induced angiogenesis with anti-vascular endothelial growth factor agents such as bevacizumab should prevent the growth of solid tumours. Choroidal metastases from lung cancer have been successfully treated with intravenous bevacizumab and chemotherapy ${ }^{7}$ and intravitreal bevacizumab with concurrent chemotherapy. 8

There have only been four case reports in the literature where patients with lung ${ }^{9}$ and breast ${ }^{10-12}$ tumours, found to have new choroidal lesions despite systemic therapy, were successfully treated with intravitreal bevacizumab.

In our cases, the choroidal metastases developed during systemic treatment; therefore, it is reasonable to presume that they were not responsive. Following intravitreal bevacizumab, visual symptoms improved and tumour regression and resolution of serous retinal detachment was obtained in all cases. The mechanism of action is potentially due to the known antiangiogenic and antipermeability properties of bevacizumab and the superior choroidal bioavailability obtained with intravitreal administration.

To the authors' knowledge, this case series is the largest reported in the literature with favourable response over a relatively long period of follow-up. Intravitreal bevacizumab can have a role in the management of choroidal metastases by reducing subretinal fluid and tumour volume, thus, preventing vision loss and improving the quality of life of patients. Further studies are warranted to confirm the efficacy of such treatment.

\section{Summary}

What was known before

- Local treatment modalities are used in the management of choroidal metastatic tumours.

- Bevacizumab is approved for carcinoma of colon/rectum.

- Few case reports of the use of intravenous and intravitreal bevacizumab have been published in the literature.

What this study adds

- Intravitreal bevacizumab can be a treatment option in choroidal metastases unresponsive to systemic chemotherapy.

- Intravitreal bevacizumab can reduce tumour volume and subretinal fluid in choroidal metastases.

- Intravitreal bevacizumab can prevent vision loss in choroidal metastatic tumours, thus, improving the quality of life of patients.

\section{Conflict of interest}

The authors declare no conflict of interest.

\section{References}

1 Bloch RS, Gartner S. The incidence of ocular metastatic carcinoma. Arch Ophthalmol 1971; 85: 673-675.

2 Camarillo C, Sànchez Ronco I, Encinas JL. Choroidal metastases. An Sist Sanit Navar 2008; 31(Suppl 3): 127-134.

3 Shields CL, Shields JA, Gross N, Schwartz GP, Lally SE. Survey of 520 eyes with uveal metastases. Ophthalmology 1997; 104: 1265-1276.

4 Chen CJ, McCoy AN, Brahmer J, Handa JT. Emerging treatments for choroidal metastases. Surv Ophthalml 2011; 56(6): 511-521.

5 Kaliki S, Shields CL, Al-Damash SA, Mashayekhi A, Shields JA. Photodynamic therapy for choroidal metastasis in 8 cases. Ophthalmology 2012; 119(6): 1218-1222.

6 Giantonio BJ, Catalano PJ, Meropol NJ, O'Dwyer PJ, Mitchell EP, Alberts SR et al. Eastern Cooperative Oncology Group Study E3200. Bevacizumab in combination with oxalilplatin, fluorouracil, and leucovorin (FOLFOX4) for previously treated metastatic colorectal cancer: results from the Eastern Cooperative Oncology Group Study E3200. J Clin Oncol 2007; 25(12): 1539-1544.

7 George B, Wirostko WJ, Conner TB, Choong NW. Complete and durable response of choroidal metastasis from nonsmall cell lung cancer with systemic bevacizumab and chemotherapy. J Thorac Oncol 2009; 4: 661-662.

8 D'Antonio C, Viterbo A, Romiti A, Maurizi Enrici M, Lauro S, Marchetti P. Complete regression of a non-small cell lung cancer choroidal metastasis with intravitreal bevacizumab. J Thorac Oncol 2012; 7: 468-469.

9 Lai CL, Fan KS, Lee YH, Chen HC, Fan WH. Intravitreal administration of bevacizumab in the treatment of choroidal metastasis in a patient with erlotinib-failed pulmonary adenocarcinoma. Lung Cancer 76 2012; 496-498.

10 Amselem L, Cervera E, Diaz-Llopis M, Montero J, Garcia-Pous M, Udaondo P et al. Intravitreal bevacizumab (Avastin) for choroidal mestastasis secondary to breast 
carcinoma: short-term follow-up. Eye (Lond) 2007; 21(4): 556-557.

11 Yao HY, Horng CT, Chen JT, Tsai ML. Regression of choroidal metastasis secondary to breast carcinoma with adjuvant intravitreal injection of bevacizumab. Acta Ophthalmol 2010; 88(7): 282-283.

12 Mansour AM, Alameddine R. Intravitreal bevacizumab for consecutive multiple choroidal breast metastatic lesions. BMJ Case Rep 2012; e-pub ahead of print 15 June 2012; doi:10.1136/bcr.03.2012.6124.
13 Rudoler SB, Corn BW, Shields CL, de Potter P, Hyslop T, Shields JA et al. External beam irradiation for choroid metastases: identification of factors predisposing to longterm sequelae. Int J Radiat Oncol Biol Phys 1997; 38(2): 2516.

14 Shields CL, Shields JA, De Potter P, Quaranta M, Freire J, Brady LW et al. Plaque radiotherapy for the management of uveal metastasis. Arch Ophthalmol 1997; 115(2): 203-209.

15 Kiratli H, Bilgic S. Transpupillary thermotherapy in the management of choroidal metastases. Eur J Ophthalmol 2004; 14(5): 423-429. 